\title{
A Simple Fuzzy Backstepping Control Method for Saucer-shaped Aircraft
}

\author{
Huali $W^{a}{ }^{a}$, Lingling Wang ${ }^{b}$, Lili Yang, Junwei Lei \\ ${ }^{1}$ Department of Control, Naval Aeronautical and Astronautical University, Yantai, 264001,China \\ aemail:tom_linhuali@126.com, bemail: lingling0711@163.com
}

Keywords: Fuzzy control, Backstepping Control; Saucer-shaped Aircraft, Stability, Lyapunov function

\begin{abstract}
A simplified linear model is studied for the complex nonlinear model for a class of saucer-shaped aircraft in this paper. Then a feedback control law is designed to achieve the tracking of given attitude angle. What is more, a kind of gain adjusting law by fuzzy changes with errors is designed in order to achieve better matching of backstepping gains. Finally, detailed analysis results of the simulation show the effectiveness of the proposed fuzzy control law.
\end{abstract}

\section{Introduction}

The saucer-shaped aircraft, which is a disc shape without a tail or rudder, can provide lift power with its whole body, and the mechanical problems caused by mass center and nozzle angle need to be considered for flight control, so the control of real-time, anti-interference and robust performance is very important [1-3]. At present, there are a lot of experts and scholars to give the control scheme. The paper [4] presents a variable structure control method for the system with a variable mass moment compounded with thrust vector. The paper [5] proposes a compound control strategy by combination of genetic algorithm and fuzzy logic based on genetic-fuzzy logic. The paper [6] uses the method of Lyapunov optimization controller, which is applied to the whole trajectory simulation of saucer-shaped aircraft. All of these methods have good robustness, but these algorithms are passive anti-interference algorithm, and the control accuracy cannot be guaranteed when the system interference is large or the change rate is high. Therefore, this paper proposes the method to use fuzzy algorithm for controller design, which can activate the anti-interference capability of the system [7-8], and meanwhile, the robustness of the system against the mismatched uncertainties is enhanced by the utilization of backstepping method. [9-11].

\section{Model Description}

According to literature [5], the ordinate dynamic model for saucer-shaped aircraft can be described as formula 1.

$$
\left\{\begin{array}{l}
M \dot{v}=-X-M g \sin \theta+P \cos (\xi+\alpha) \\
M v \dot{\theta}=Y-M g \cos \theta+P \sin (\xi+\alpha) \\
J_{z}^{\prime} \dot{\omega}_{d b z}^{b}=X_{P} P \sin \xi+y_{T} P \cos \xi+M_{z}+M_{z}\left(x_{b}\right)-\mu P \sin \xi \cdot x_{b} \\
\dot{\vartheta}=\omega_{d b z}^{b}
\end{array}\right.
$$

Theorem1: Assume the linear approximation system of nonlinear system is asymptotically stable, and then any linear feedback that can make the linear approximation system stable can also asymptotically stabilize the original nonlinear system, and at least make the nonlinear system locally stable.

The linear dynamic model of missile is studied in literature [12]. Similarly, we can also study the linear approximation system of nonlinear system as described in formula 1, in order to find the control law which can make the original nonlinear system asymptotically stable. According to the literature [5], the linear approximation system for the system as described in formula 1 can be presented in formula 2. 


$$
\left\{\begin{array}{l}
\dot{\omega}_{z}=-a_{24} \theta+a_{24} \vartheta+a_{25} x_{b}+a_{z}^{\prime} \xi \\
\dot{\theta}=\left(a_{33}-a_{34}\right) \theta+a_{34} \vartheta+a_{x} \xi \\
\dot{\vartheta}=\omega_{z}
\end{array}\right.
$$

The definitions for all the symbols in formula 2 can be found in reference [2].

The transfer function can be derived from the above state equation, and this is a non-minimum phase system, as described in formula 3 , where $a_{z}<0, a_{34}>0, a_{24}>0, a_{x}>0$.

$$
G_{\xi}^{\vartheta}=\frac{a_{z}\left(S+a_{34}\right)-a_{x} a_{24}}{S\left[S^{2}+a_{34} S-a_{24}\right]}
$$

\section{Simple Fuzzy Backstepping Mode Controller Design}

Define an error variable as $z_{1}=\vartheta-\vartheta^{*}$.

Then we get

$$
\dot{z}_{1}=\dot{\vartheta}-\dot{\vartheta}^{*}=\omega_{z}
$$

Design the desired acceleration signal $\omega_{z}^{d}$ as

$$
\omega_{z}^{d}=-k_{1} z_{1}
$$

Then its derivative can be written as

$$
\dot{\omega}_{z}^{d}=-k_{1} \dot{z}_{1}=-k_{1} \omega_{z}
$$

Define an angular velocity error variable as $z_{2}=\omega_{z}-\omega_{z}^{d}$.

Then we get

$$
\dot{z}_{2}=\dot{\omega}_{z}-\dot{\omega}_{z}^{d}=-a_{24} \theta+a_{24} \vartheta+a_{25} x_{b}+a_{z}^{\prime} \xi-\dot{\omega}_{z}^{d}
$$

Choose an auxiliary control variable as

$$
u=a_{25} x_{b}+a_{z}^{\prime} \xi
$$

Then design the backstepping control law as

$$
u=-\left(-a_{24} \theta+a_{24} \vartheta\right)-k_{1} \omega_{z}-k_{2} z_{2}
$$

Fuzzy laws are established to design proper backstepping parameters $k_{1}$ and $k_{2}$ Choose the initial gain values as $k_{10}=5, k_{20}=25$. If $|e|>0$, then $k_{1 a}$ and $k_{2 a}$ should increase the value.

And $k_{1}=k_{0}+k_{1 a}, k_{2}=k_{20}+k_{2 a}$.

Design the fuzzy system with input $e$, and output $k_{1 a}$ and $k_{2 a}$.

Definitions of fuzzy set for inputs and outputs are described as the following.

$$
\begin{aligned}
& e=\left\{\begin{array}{lllll}
N B & N M & Z O & P M & P B
\end{array}\right\} \\
& k_{1 a}=\left\{\begin{array}{lllll}
N B & N M & Z O & P M & P B
\end{array}\right\} \\
& k_{1 a}=\left\{\begin{array}{lllll}
N B & N M & Z O & P M & P B
\end{array}\right\}
\end{aligned}
$$

And then define a medium error value as $e_{m}=5 / 57.3, k_{1 a m}=30$, and $k_{2 a m}=140$. Then the membership function can defined as below figure 1 to figure 3 .

Define the fuzzy law of $\Delta k_{1}$ and $\Delta k_{2}$ as the following:

$\mathrm{R} 1:$ IF $|e|$ is $\mathrm{PB}$ Then $\Delta k_{1}$ is $\mathrm{PB}$ and $\Delta k_{2}$ is $\mathrm{PB}$

$\mathrm{R} 2: \quad \mathrm{IF}|e|$ is $\mathrm{PM}$ Then $\Delta k_{1}$ is $\mathrm{PM}$ and $\Delta k_{2}$ is $\mathrm{PM}$

R3: IF $|e|$ is ZO Then $\Delta k_{1}$ is $\mathrm{ZO}$ and $\Delta k_{2}$ is $\mathrm{ZO}$ 


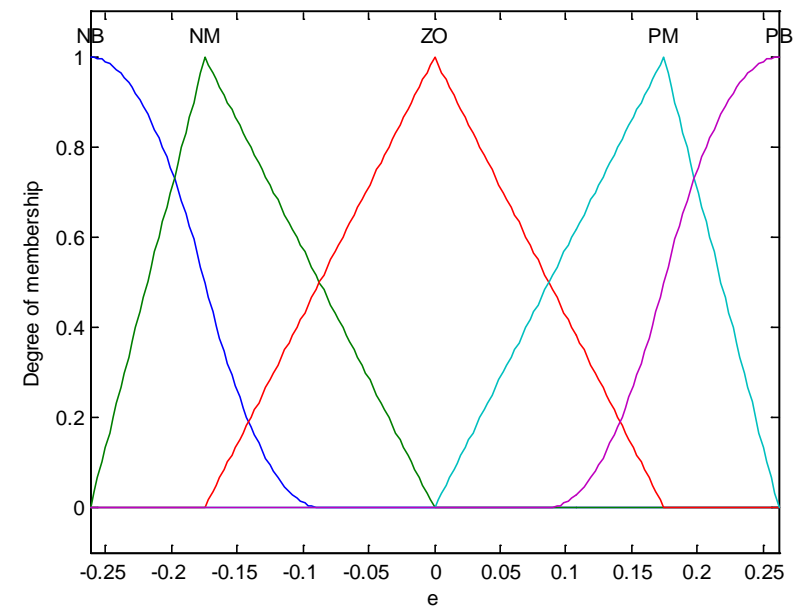

Figure 1 Membership function of erro

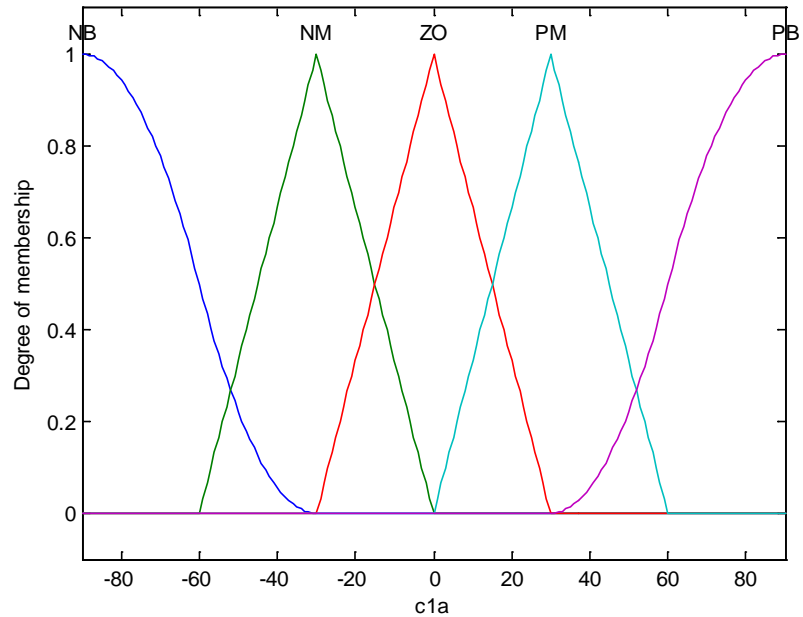

Figure 2 Membership function of $k_{1}$

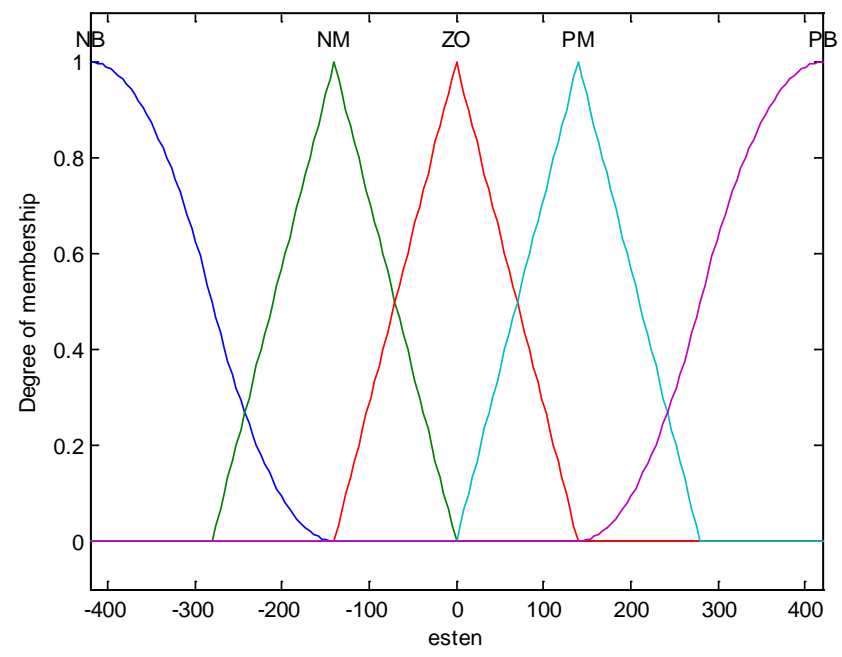

Figure 3 Membership function of $k_{2}$

\section{Simulation Analysis}

To testify the rightness and effectiveness of the above fuzzy backstepping control method, numerical simulation was done and model parameters can see below Table 1. Figure 4 Shows the attitude angle of aircraft and we can find that the rise time is only $0.1 \mathrm{~s}$, it is a very quick speed for 
saucer shaped aircraft. Figure 5 show that no mass control was used and Figure 8 shows the jet control curve. Figure 6 and Figure 7 shows the adjustment of control gain K1 and K2. All above curve testified that the proposed fuzzy backstepping control method was effective and reasonable. So it has a high value for engineering application.

Table 1 Kinetic coefficients

\begin{tabular}{|c|c|c|c|c|c|}
\hline$a_{24}$ & $a_{25}$ & $a_{34}$ & $a_{37}$ & $a_{x}$ & $a_{z}\left(a_{z}^{\prime}\right)$ \\
\hline 829.0773 & -19.1620 & 2.3230 & 0.0013 & 0.0289 & -12.8979 \\
\hline
\end{tabular}

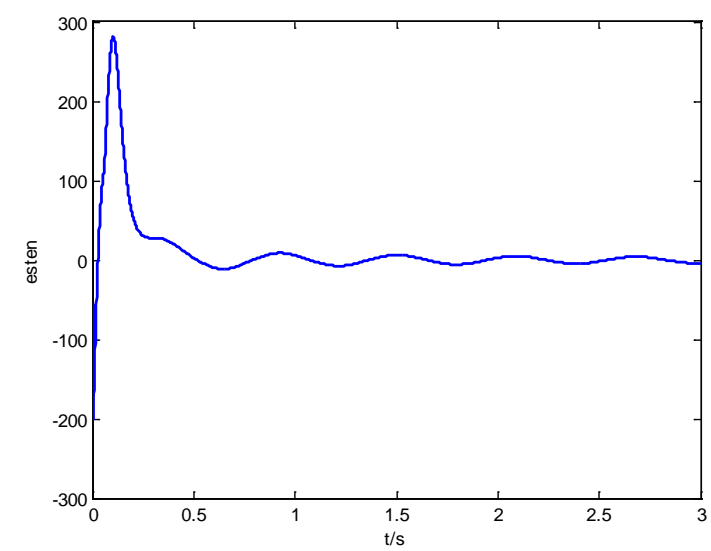

Figure 4 Curve of attitude angle

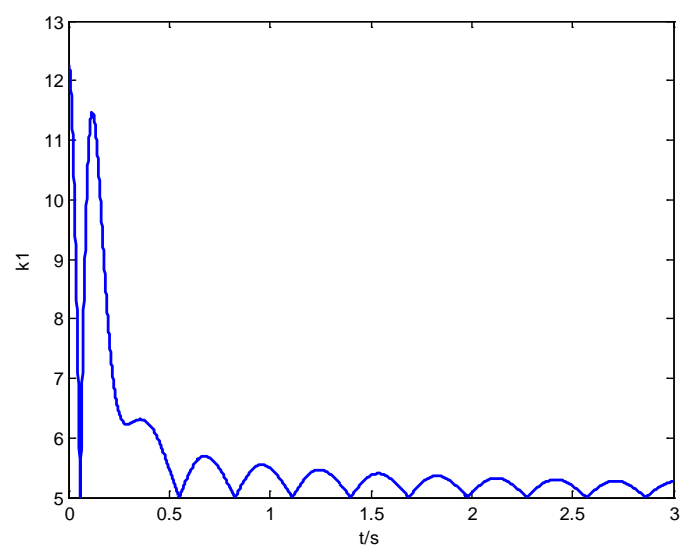

Figure 6 Curve of coefficient $k_{1}$

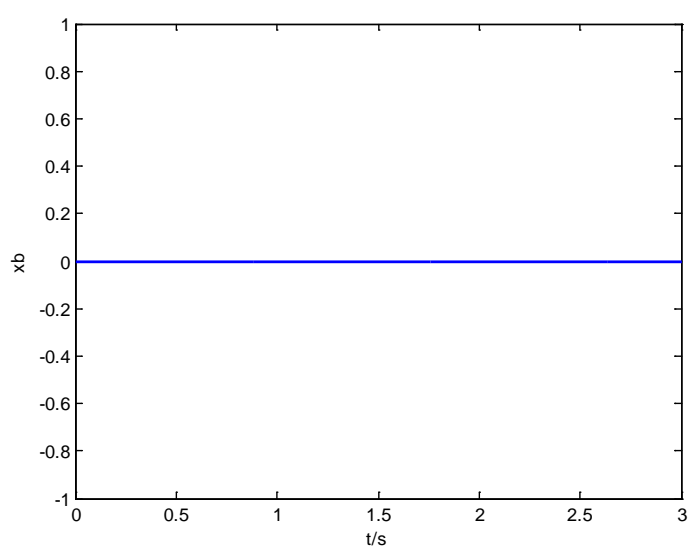

Figure 5 Curve of Mass control

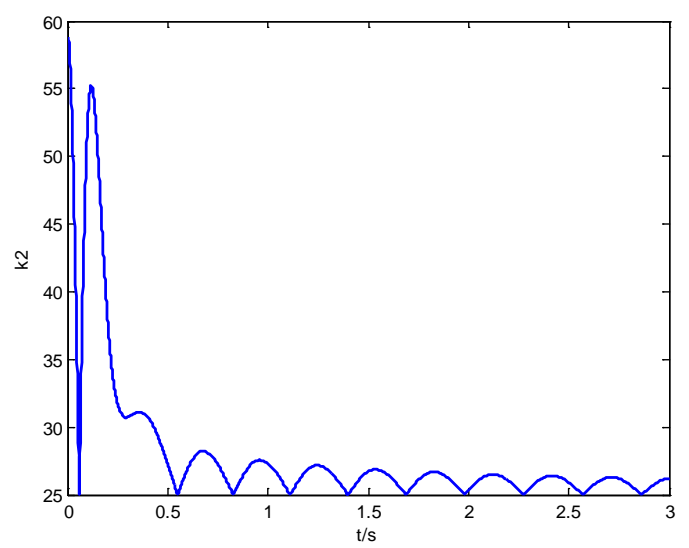

Figure7 Curve of coefficient $k_{2}$

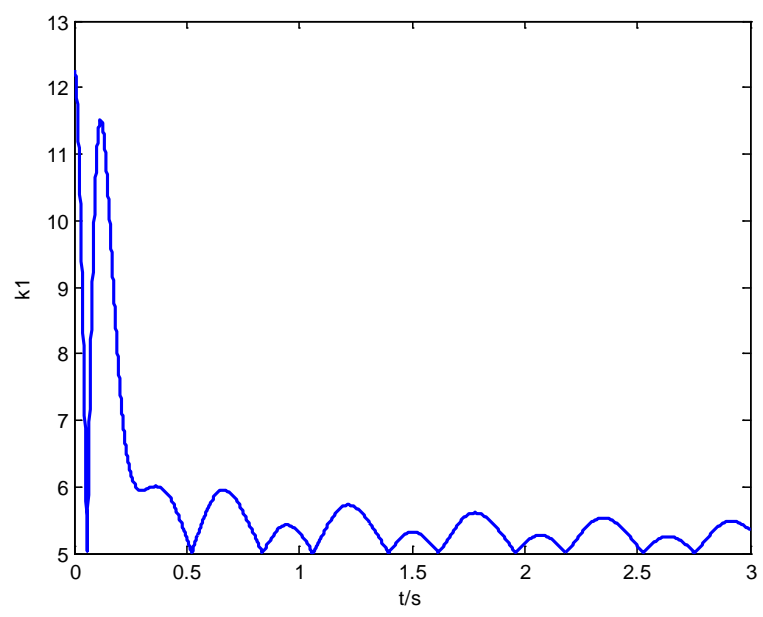

Figure8 Curve of Jet Control $\xi$ 


\section{Conclusions}

This paper proposes a fuzzy backstepping control algorithm based on the simplified longitudinal channel model of the saucer-shaped aircraft. The simulation results show that compared with the backstepping control method, the algorithm proposed in this paper has a more simplified control law expression and better anti-interference performance, and meanwhile with less requirements for model precision .

\section{Reference}

[1] Gu Wenjin, Yang Kan, Research on GPC the Saucer -like Air Vehicle by GPC [J]. Computer Simulation, 2011, 28(3):91-96(in Chinese).

[2]Yu Junli, Wang Linlin, GaoGe. Using wing tip devices to improve performance of saucer-shaped aircraft [J]. Chinese Journal of Aeronautics, 2006, 19(4):309-314.

[3] Ma Songhui, Wu Chengfu, Chen Huaimin. Study on stability and manoeuvrability of flying wing aircraft [J]. Flight Dynamics, 2006, 24(3):18-19(in Chinese).

[4] Peng Shanguo, Yang Kan, Gu Wenjin. Research on Compound Control Method of Saucer-like Air Vehicle Based on VSC [J]. 2010, 27(11):32-40(in Chinese).

[5] Wu Jinhuang, Xu Sheng-hong, Gu Wenjin, Distribution Strategy based on Genetic-Fuzzy Logic for Compound Control of Flying Saucer[J]. Fire Control and Command Control, 2008, 33(5):63-65 (in Chinese).

[6] Gu Wenjin, Ma Mingzhong, Design of Controllers for Flying Saucer Based on Lyapunov Optimization[J]. Flight Dynamics, 2008, 26(5):25-27 (in Chinese).

[7]L Yang, W Zhang, D Huang, J Che. Adaptive Fuzzy Backstepping Control for Underactuated Quadrotor UAV [J]. Xibei Gongye Daxue Xuebao/journal of Northwest Polytechnical University, 2015, 33(3):495-499(in Chinese).

[8]Liu Baiting, Wu Yun-jie, Huang Yanfu, Research of Adaptive Backstepping Sliding Mode Controller Based on Fuzzy Disturbance Observer[J]. Journal of System Simulation, 2011, 23(8):1677-1680(in Chinese).

[9] Ashfaq Ahmad Mian, Modeling and Backstepping-based Nonlinear Control Strategy for a 6 DOF Quadrotor Helicopter[J]. Chinese Journal of Aeronautics, 2008, 21(3):261-268.

[10] Zhang Chao, Chen ZongJi, Wei Chen, Sliding mode disturbance observer-based backstepping control for a transport aircraft[J]. Science China (Information Sciences), 2014, 57(5):1-16.

[11] Ran Maopeng, Wang Qing, Hou Delong, Dong Chaoyang, Backstepping design of missile guidance and control based on adaptive fuzzy sliding mode control[J]. Chinese Journal of Aeronautics, 2014, 27(3):634-642.

[12] Wu Sentang, Fei Yuhua, Flight control systems [M]. Beijing: Beijing University of Aeronautics and Astronautics Press, 2005:73-88(in Chinese). 